\title{
Calculating the Exact Experimental Density of the Dark Energy in the Cosmos Assuming a Fractal Speed of Light
}

\author{
Mohamed S. El Naschie \\ Department of Physics, Faculty of Science, University of Alexandria, Alexandria, Egypt \\ Email: Chaossf@aol.com
}

Received 2 December 2013; revised 2 January 2014; accepted 9 January 2014

Copyright (c) 2014 by author and Scientific Research Publishing Inc.

This work is licensed under the Creative Commons Attribution International License (CC BY).

http://creativecommons.org/licenses/by/4.0/

(c) (i) Open Access

\begin{abstract}
Fractal speed of light theory is a variation of Magueijo-Smolin varying speed of light (VSL) theoretical modification of Einstein's energy mass relation. We use this theory to derive an exact value for the missing dark energy which is found to be in astonishing agreement with the latest result of the WMAP measurement and the independent supernova analysis. Thus while Einstein's formula predicts $95.5 \%$ more energy than found in highly precise astrophysical measurement, our VSLbased calculation indicates an exact theoretical value of only $4.508497 \%$ real energy. Consequently, the exact conjectured missing dark energy must be $95.491502 \%$. By any standards, this is an astounding confirmation for both the cosmological measurement and the VSL theory.
\end{abstract}

\section{Keywords}

Nonlinear Dynamics; Fractals; Dark Energy; Quantum Gravity; Varying Speed of Light Theory

\section{Introduction}

In the present work, we use a fractal speed of light theory to resolve the issue of the missing dark energy in the cosmos which has become a colossal problem threatening the very foundations of theoretical physics and cosmology [1] [2]. There is new undeniable confirmation via sophisticated analysis of WMAP and supernova measurement that approximately $95.5 \%$ of the energy which the cosmos should contain is not there to see or measure [1]. It should really go without saying that at this level of analysis, we are lumping dark energy and dark matter in one category for a start which is the quintessence of Einstein's insight [1]-[10].

In this work which constitutes a concerted effort of various avant-garde theories brought to bear on the subject of dark energy, we give an exact analysis showing that the energy value predicted by Einstein's special theory of 
relativity $E=m_{o} c^{2}$, where $E$ is the energy, $m_{o}$ is the rest mass and $c$ is the velocity of light gives the wrong answer when it comes to calculating the intergalactic energy scales [1] [2]. The correct equation in this case must take quantum mechanics and nonclassical topology of the fabric of spacetime into account [2]-[8] in order to bring theoretical predictions and experimental measurement together. To this end we will use the equation developed by Magueijo and Smolin [9] [10] to modify Einstein's relativity theory using a fractal version of the theory of varying speeds of light (VSL). Subsequently we give the VSL equation a novel topological interpretation using an eleven dimensional theory akin to that of super gravity and M-theory but within a fractal setting [7] [8]. At the end, the obtained result confirms the absence of $95.4915 \%$ of the energy of the cosmos dubbed dark matter and dark energy [1]-[3] as well as the experimental findings based on the WMAP and super nova measurement which indicates $95.5 \%$ dark matter and dark energy [1] [2]. The amazing agreement between theory and experiment is unparalleled and leaves very little room for doubting the cosmological measurement [1] or the VSL theory [9] [10] or indeed for that matter, the extra dimensions and fractal nature of spacetime at the quantum scale [3]-[18]. In fact contemplating the entire big picture starting from F. Zwieky's observation as well as the confirmed increased acceleration of cosmic expansion we must conclude the existence of attracting dark matter and repulsing dark energy. Similarly, these measurements [1] [2] and experiments [19]-[30] go a long way towards heralding the physical reality of cosmic entanglement, Hawking negative energy fluctuation as well as Rindler and Unruh effects [29].

\section{The Energy-Mass Relation of the VSL Theory}

The completely new idea behind the derivation of the energy-mass relationship using the VSL theory basically comes from Nottale's theory of scale relativity [3]. Both scale relativity and VSL theory [9] [10] take the Planck energy $E_{p}=1 / L_{p}$ where $L_{p}$ is the Planck length as invariant. Consequently J. Magueijo and L. Smolin arrive at their replacement of Einstein's single equation $E=m c^{2}$ by three remarkable and thoroughly ingenious equations which due to space limitation are given here without elaboration [9]:

$$
E_{o}=\frac{m_{o} c^{2}}{1+\frac{m_{o} c^{2}}{E_{p}}}
$$

with the usual transformation $m=\gamma m_{o}$ where $\gamma$ is the Lorentz factor but with $E$ and momentum $p$ transforming as [9]

$$
E=\frac{m}{1+\frac{m}{E_{p}}}
$$

and

$$
P=\frac{m v}{1+\frac{m}{E_{p}}}
$$

The preceding equations which are explained lucidly in the original paper of Mageuijo and Smolin [9] [10] give us the possibility of admitting a ratio $m_{o} c^{2} / E_{P}$ far larger than unity. In other words through the postulates of VSL theory $\mathrm{m}_{\mathrm{o}} \mathrm{c}^{2}$ can become many times larger than $E_{p}$ without violating Lorentzian invariance, nor the $1 / L_{p}$ invariance of the theory [9]. To show that this superficially contradictory statement leads to a true formula for quantum relativity and gives results in full agreement with the cosmological measure is our next task.

\section{Dark Energy for the Extra Dark Dimensions}

From the first equation of Magueijo-Smolin [9] [10] it is clear that our crucial ratio $m_{o} c^{2} / E_{P}$ could take three vital values:

$$
m_{o} c^{2} / E_{P} \cong 0
$$

i.e. we have Einstein's classical equation of special relativity. 


$$
m_{o} c^{2} / E_{P}=1
$$

i.e. we have the Newtonian expression for energy when setting $c=v$ and find $E=(1 / 2) m v^{2}$.

$$
m_{o} c^{2} / E_{P}=21
$$

This is a crucial situation for us as we will explain in a moment. The energy in this case could be written as

$$
E_{o}=\frac{m_{o} c^{2}}{1+21}=\frac{m_{o} c^{2}}{22} .
$$

Let us consider the above from the view point of spacetime dimensions [7] [8] and in particular from the view point of the theories involving extra spacetime and compactified dimensions [7]-[18].

In case (a) above we could say that spacetime fuses into $3+1=4$ dimensions. There are zero hidden dimensions in this theory. The total energy $E_{o}$ is not reduced or diluted by extra dimensions, which is behind any fractal spacetime theory at least implicitly [8] [11] [23].

In case (b) above we could say, somewhat surprisingly, that we have a hidden dimension, namely time appearing only as a parameter. The energy $m c^{2} \rightarrow m v^{2}$ is divided therefore by 2 .

In the most important case (c) above however we could interpret the factor 21 as the number of dark dimensions of 26 bosonic string theory [7] [8] [17] when a 5 dimensional Kaluza-Klein theory is assumed [21] [22]. Alternatively we take $1+21=22$ factor to mean the 22 compactified [17] invisible, dark and hidden dimensions of non-super symmetric string theory [13] [15] [17]. In fact the 22 dark dimensions could be interpreted differrently as 11 bosons and 11 fermions of a super symmetric 11 dimensional theory [11]-[13]. That way we have a new modified energy-mass relation almost identical to Einstein's equation with the exception that the energy is now reduced by a factor of 1/22. This reduction amounts to diluting or spreading thinly Einstein's energy because of the extra dimensions which exist but were not taken into account in the derivation of the 4D theory of Einstein. Therefore we can write [9]

$$
E_{\max }=\left(\frac{1}{22}\right)\left(m_{o} c^{2}\right) \cong(0.04545) m_{o} c^{2} .
$$

This result is tantamount to saying that $(1-0.04545)(100)=95.454 \%$ of the energy in the universe is dark energy. The result is very close indeed to the experimental measurement of $95.5 \%$ [1] and means that the extra dark extra dimension of micro spacetime is the cause for dispersing energy to the extent that we notice only a fraction equal to $4.5 \%$ of the classically predicted total. It is not difficult to convince oneself of the intimate relation between symmetries, dimensions, degrees of freedom and $E$. Noether's theorem [16] on the laws of conservation including of course energy, is behind our result [16] [22]-[24]. On the other hand we know that fractal spacetime implies extra dark dimensions as well as nonclassical microscopic mechanics which we perceive as quantum mechanics [4]-[6]. Thus we will look once more at the preceding result from the view point of quantum mechanics and thus quantum relativity, i.e. quantum gravity [3]-[5] [13] [15]-[17].

\section{Hardy's Quantum Entanglement Behind Dark Energy}

The classical Heterotic string theory [17] stipulates three dimensions, namely 26, 16 and 26-16=10 superstring dimensions of which 6 are compactified to give a 4 dimensional classical or relativistic spacetime [18]. Setting this theory in fractal dressing one finds $26+\mathrm{k}, 16+\mathrm{k}$ and $26+k-(16+k)=10$ where $k=0.18033989$. Thus our hidden dark dimensions of the previous analysis are made "fractally" accurate by writing [7] [8] [21]-[30]

$$
26+k-4=22+k
$$

where $k=\phi^{3}\left(1-\phi^{3}\right)=0.18033989$ and $\phi=(\sqrt{5}-1) / 2=0.618033989$. Since the hidden dimensions reduce the energy density as explained in the previous section we see that the exact energy $E_{\max }$ must be logically divided by $22+\mathrm{k}$ and consequently [21]-[25]

$$
E=\left(\frac{1}{22+k}\right)\left(m_{o} c^{2}\right)=\frac{1}{2}\left(\phi^{5}\right)\left(m_{o} c^{2}\right)
$$

The remarkable thing is that $\phi^{5}$ is the exact theoretical value which Hardy found for a generic quantum en- 
tanglement [19]. This value was also experimentally confirmed with high precision and sophisticated quantum informational methods using modern beam splitters etc. [5] [6] [19] [20].

The same previous result can be found directly from delicate light cone quantized Lorentzian transformation [18] and confirms the intuitive initial hunch that $E=(1 / 2)\left(\phi^{5}\right) m_{o} c^{2}$ is a quantum gravity formula for quantum relativity [22]. Consequently we could rephrase our hidden dark dimensions interpretation [7] [14] [15] to mean the inclusion of quantum entanglement which is clearly the cause for the $95.5 \%$ reduction in the classical energy prediction and therefore give a quantum gravity explanation for the missing dark energy [12]. The reader is reminded again that the $95.5 \%$ include dark energy as well as dark matter converted to energy [1] [23]. There are many other subtle points which need not discussed here in details regarding the topological speed of light being $\phi$ and the three dimensional topological mass of a quantum particle $m=\phi^{3}$ which leads via the classical kinetic energy formula $E=\frac{1}{2} m v^{2}$ to $E=\left(\frac{1}{2}\right)\left(\phi^{3}\right)\left(\phi^{2}\right)=\left(\phi^{5} / 2\right)$ in full agreement with the above when setting $\left(m_{o} c^{2}\right)=1$. This fascinating topological unit interval Cantorian spacetime approach is expanded upon in Reference [21]-[25].

\section{Conclusion}

We give a hidden dark dimension interpretation to the Magueijo-Smolin energy mass equation of VSL theory [9] [10]. The quantitative result fully explains the missing dark energy of the cosmos. Einstein's equation predicts $E=m_{o} c^{2}$. The VSL equation on the other hand predicts approximately $1 / 22$ of this value. Accurate calculation shows that $22=26-4$ hidden dark dimensions of bosonic string theory should be 22.18033989 when taking the "fine structures" of the strings into account and consequently the exact reduction is not $1 / 22$ but $1 /(22+k)=\phi^{5} / 2$ where $\phi=(\sqrt{5}-1) / 2$. On the other hand, Hardy's quantum generic entanglement is given exactly by $\phi^{5}$, a quantity which was found experimentally [5] [6] [19]. Consequently the reduction factor in the energy which is the cause for suspecting a $95.5 \%$ missing dark energy in the cosmos is clearly due to the inclusion of the quantum entanglement effect in Einstein's formula to elevate it from a relativistic formula to a quantum relativity formula predicting accurately the real energy content of the cosmos. Either way the rationale behind the dark dimensions and the quantum entanglement interpretation is the same. Many infinite gaps in the fabric of spacetime itself are what simulates quantum entanglement or hidden dark dimensions reduction of the total energy predicted by the classical formula and that by as much as $95.5 \%$ [1] [2]. At this point, two further remarks are in order. First, the relation between internal symmetries of spacetime, extra compacted dimension and energy is not entirely surprising in view of Noether's theorem [23]. Second, the difference between dark energy and dark matter not considered in the present work is intimately related to the sum of quasi dimensional inverse coupling which was touched upon in a previous publication [24]. The present result confirms that the cosmological measurements are accurate and fully deserve the 2011 Nobel Prize and that VSL theory is a valid and accurate theory [9] [10]. Similar ideas within a limited context compared to the present paper maybe found in Reference [26]. For a short review of work related to the present paper, we recommend Reference [27]-[30]. Finally, we draw attention to new result which we perceive as tantalizing, because it raise the real possibility that Hawking radiation, Unruh temperature, and Rindler wedge are as physically real as cosmic expansion being linked to it [29].

\section{References}

[1] Amendola, L. and Tsujikawa, S. (2010) Dark Energy: Theory and Observations. Cambridge University Press, Cambridge. http://dx.doi.org/10.1017/CBO9780511750823

[2] Baryshev, Y. and Teerikorpi, P. (2002) Discovery of Cosmic Fractals. World Scientific, Singapore.

[3] Nottale, L. (2011) Scale Relativity. Imperial College Press, London.

[4] Ord, G. (1983) Fractal Space-Time: A Geometric Analogue of Relativistic Quantum Mechanics. Journal of Physics A: Mathematical and General, 16, 1869. http://dx.doi.org/10.1088/0305-4470/16/9/012

[5] El Naschie, M.S. (2011) Quantum Entanglement as a Consequence of a Cantorian Micro Spacetime Geometry. Journal of Quantum Information Science, 1, 50-53. http://www.SCRIP.org/journal/jqis http://dx.doi.org/10.4236/jqis.2011.12007

[6] He, J.-H., et al. (2011) Quantum Golden Mean Entanglement Test as the Signature of the Fractality of Micro Space- 
time. Nonlinear Science Letters B, 1, 45-50.

[7] El Naschie, M.S. (2009) The Theory of Cantorian Spacetime and High Energy Particle Physics (An Informal Review). Chaos, Solitons \& Fractals, 41, 2635-2646. http://dx.doi.org/10.1016/j.chaos.2008.09.059

[8] El Naschie, M.S. (2004) A Review of E-Infinity Theory and the Mass Spectrum of High Energy Particle Physics. Chaos, Solitons \& Fractals, 19, 209-236. http://dx.doi.org/10.1016/S0960-0779(03)00278-9

[9] Magueijo, J. and Smolin, L. (2002) Lorentz Invariance with an Invariant Energy Scale. Physical Review Letters, 88, Article ID:190403.

[10] Magueijo, J. (2003) Faster than the Speed of Light. William Heinemann, London.

[11] El Naschie, M.S. (2006) On an Eleven Dimensional E-Infinity Fractal Spacetime Theory. International Journal of Nonlinear Sciences and Numerical Simulation, 7, 407-409.

[12] El Naschie, M.S. (2006) The “Discrete” Charm of Certain Eleven Dimensional Spacetime Theory. International Journal of Nonlinear Sciences and Numerical Simulation, 7, 477-481.

[13] Duff, M. (1999) The World in Eleven Dimensions. IOP Publishing Ltd., Bristol.

[14] Yau, S.T. and Nadis, S. (2010) The Shape of Inner Space. Basic Book, Persens Group, New York.

[15] Randal, L. (2005) Warped Passages. Allen Lane-Penguin Books, London.

[16] Penrose, R. (2004) The Road to Reality. Jonathan Cape, London.

[17] Becker, K., Becker, M. and Schwarz, J. (2007) String Theory and M-Theory. Cambridge University Press, Cambridge.

[18] Schwarz, P.M. and Schwarz, J.H. (2004) Special Relativity from Einstein to Strings. Cambridge University Press, Cambridge.

[19] Hardy, L. (1993) Nonlocality of Two Particles without Inequalities for almost All Entangled States. Physical Review Letters, 71, 1665-1668.

[20] Bengtsson, I. and Zyczkowski, K. (2008) Geometry of Quantum States. Cambridge University Press, Cambridge.

[21] El Naschie, M.S. (2013) What Is the Missing Dark Energy in a Nutshell and the Hawking-Hartle Quantum Wave Collapse. International Journal of Astronomy and Astrophysics, 3, 205-211.

[22] Marek Crnjac, L. and El Naschie, M.S. (2013) Quantum Gravity and Dark Energy Using Fractal Planck Scaling. Journal of Modern Physics, 4, 31-38.

[23] Helal, M.A., Marek-Crnjac, L. and He. J.-H. (2013) The Three Page Guide to the Most Important Results of M. S. El Naschie's Research in E-Infinity Quantum Physics and Cosmology. Open Journal of Microphysics, 3, 141-145.

[24] El Naschie, M.S. (2007) From Symmetry to Particles. Chaos, Solitons \& Fractals, 427-430. http://dx.doi.org/10.1016/j.chaos.2006.09.016

[25] El Naschie, M.S. (2013) A Unified Newtonian-Relativistic Quantum Resolution of the Supposedly Missing Dark Energy of the Cosmos and the Constancy of the Speed of Light. International Journal of Modern Nonlinear Theory and Application, 43-54.

[26] El Naschie, M.S. (2013) Using Varying Speed of Light Theory to Elucidate and Calculate the Exact Experimental Percentage of the Dark Energy in the Cosmos. Fractal Spacetime and Noncommutative Geometry in Quantum and High Energy Physics, 3, 35-38.

[27] He, J.-H. and Marek-Crnjac, L. (2013) The Quintessence of El Naschie's Theory of Fractal Relativity and Dark Energy. Fractal Spacetime and Noncommutative Geometry in Quantum and High Energy Physics, 3, 130-137.

[28] Marek-Crnjac, L. and He. J. (2013) The Three Page Guide to the Most Important Results of M. S. El Naschie’s Research in E-Infinity Quantum Physics and Cosmology. International Journal of Astronomy and Astrophysics, 464-471.

[29] El Naschie, M.S. (2013) A Rindler-KAM Spacetime Geometry and Scaling the Planck Scale Solves Quantum Relativity and Explains Dark Energy. International Journal of Astronomy and Astrophysics, 483-493.

[30] El Naschie, M.S. (2013) Electromagnetic and Gravitational Origin of Dark Energy in Kaluza-Klein D = 5 Spacetime. PIERS Proceeding, Stockholm, Sweden, 91-97. 